\title{
Video Article \\ Using Facial Electromyography to Assess Facial Muscle Reactions to Experienced and Observed Affective Touch in Humans
}

\author{
Anbjørn Ree ${ }^{1}$, India Morrison ${ }^{2}$, Håkan Olausson ${ }^{2}$, Uta Sailer ${ }^{1}$, Markus Heilig ${ }^{2}$, Leah M. Mayo ${ }^{2}$ \\ ${ }^{1}$ Department of Behavioral Sciences in Medicine, Institute of Basic Medical Sciences, Faculty of Medicine, University of Oslo \\ ${ }^{2}$ Center for Social and Affective Neuroscience, Department of Clinical and Experimental Medicine, Linköping University
}

Correspondence to: Leah M. Mayo at leah.mayo@liu.se

URL: https://www.jove.com/video/59228

DOI: doi: $10.3791 / 59228$

Keywords: Behavior, Issue 145, facial electromyography, C-tactile afferents, affective touch, affect, emotion, corrugator

Date Published: 3/15/2019

Citation: Ree, A., Morrison, I., Olausson, H., Sailer, U., Heilig, M., Mayo, L.M. Using Facial Electromyography to Assess Facial Muscle Reactions to Experienced and Observed Affective Touch in Humans. J. Vis. Exp. (145), e59228, doi:10.3791/59228 (2019).

\section{Abstract}

"Affective" touch is believed to be processed in a manner distinct from discriminatory touch and to involve activation of C-tactile (CT) afferent fibers. Touch that optimally activates CT fibers is consistently rated as hedonically pleasant. Patient groups with impaired social-emotional functioning also show disordered affective touch ratings. However, relying on self-reported ratings of touch has many limitations, including recall bias and communication barriers. Here, we describe a methodological approach to study affective responses to touch via facial electromyography (EMG) that circumvents the reliance on self-report ratings. Facial EMG is an objective, quantitative, and non-invasive method to measure facial muscle activity indicative of affective responses. Responses can be assessed across healthy and patient populations without the need for verbal communication. Here, we provide two separate datasets demonstrating that CT-optimal and non-optimal touch elicit distinct facial muscle reactions. Moreover, facial EMG responses are consistent across stimulus modalities, e.g. tactile (experienced touch) and visual (observed touch). Finally, the temporal resolution of facial EMG can detect responses on timescales that supersede that of verbal reporting. Together, our data suggest that facial EMG is a suitable methodology for use in affective tactile research that can be used to supplement, or in some cases, supplant, existing measures.

\section{Video Link}

The video component of this article can be found at https://www.jove.com/video/59228/

\section{Introduction}

C-tactile (CT) afferents are proposed to convey the affective component of touch, which can be distinguished from the discriminative aspects of touch processed via $A \beta$ fibers ${ }^{1,2}$. CT-mediated affective touch is believed to play an integral role in social affiliative behaviors ${ }^{3}$, leading to the "skin as a social organ" hypothesis ${ }^{4}$. Physical ${ }^{5,6}$, developmental ${ }^{7}$, and psychiatric ${ }^{8,9}$ factors can influence CT-mediated touch processing. Thus, establishing an objective measure to quantify affective reactions to CT-relevant touch is critical to allow for comparisons across populations.

In recent years, much insight has been gained regarding the characteristics of CT afferents. These unmyelinated afferents demonstrate an inverted U-shaped firing frequency, with velocities of $1-10 \mathrm{~cm} / \mathrm{s}$ ("CT-optimal") eliciting the greatest frequency and both greater ("fast nonoptimal") or lesser ("slow non-optimal") velocities eliciting reduced firing ${ }^{10}$. CT firing frequency correlates with self-reported ratings of touch "pleasantness", producing a similar inverted U-shaped curve in pleasantness ratings ${ }^{10}$. Moreover, CT-afferents also respond most robustly to stimuli close to skin temperature ${ }^{11}$. These fibers also show distinct conduction speeds. The unmyelinated CT afferents are slower ${ }^{2}$ and thus the volley of afferent input to the cortex shows a temporal lag when compared to the speed of the faster, myelinated $A \beta$ fibers ${ }^{1,12}$. Affective and discriminative touch can also be distinguished on a neural level. While both types of touch activate overlapping somatosensory areas, affective touch is more likely to activate the posterior insula, while discriminative touch activates sensorimotor areas ${ }^{13,14,15,16}$. This activation pattern is consistent whether the touch is directly experienced or merely observed ${ }^{17}$, suggesting that affective touch is not just a "bottom-up" process driven by physical activation of CT afferents, but also involves "top-down" integration of multimodal sensory processing.

Situations in which CT processing is deficient or otherwise atypical has also provided insight into the functional significance of these afferents. In a unique patient group with a heritable mutation affecting the nerve growth factor $\beta$ gene, there is a reduction in the density of thin and unmyelinated nerve fibers, including CT afferents. Compared to healthy controls, these patients report touch at CT-optimal velocities as less pleasant ${ }^{5}$. The converse scenario is also true; patients who lack myelinated $A \beta$ fibers are able to retain a faint sensation of pleasant touch carried by the still intact CT afferents ${ }^{6}$. Abnormal affective touch processing is not just confined to instances of physical changes in CT-afferents. Across patient and healthy populations, those higher on the spectrum of autistic traits reported reduced pleasantness ratings of touch ${ }^{8}$. Psychiatric patients also demonstrate reduced hedonic ratings of affective touch, with a history of childhood maltreatment as one of the most consistent predictors of dysregulated affective touch awareness ${ }^{8}$. Dysregulation in the CT-based affective touch system in anorexia nervosa has also been reported $^{9}$. Thus, both physical and psychological factors can influence affective touch processing, and as such, it is imperative to establish methodologies that can be applied to all individuals in an equitable and comparable manner. 
Insights into normo-typical and dysregulated affective processing have the opportunity to provide a more nuanced picture of many patient groups. However, one potential limitation of affective touch research is the necessity of self-reported ratings. At times, self-report can be unreliable ${ }^{18}$ and subject to recall bias ${ }^{19}$. Inquiries of self-report can psychologically remove a participant from the current setting, limiting the ecological validity of the responses and removing them temporally from the experience ${ }^{20}$. Moreover, self-report relies on a firm understanding of language and semantics, making cross-cultural and developmentally diverse (e.g. infant and toddler-aged individuals) comparisons challenging. For instance, individuals with an autism spectrum diagnosis frequently show distinct behavioral responses to touch ${ }^{21}$, but can also have difficulties in communicating verbally ${ }^{22}$. Thus, finding non-invasive methods to measure responses to touch that circumvent a reliance on selfreport may translate, at least, to a better understanding of the mechanisms of affective touch, and at most, novel insights into dysregulation of social processing in patient populations.

Facial electromyography (EMG) is a suitable candidate to objectively assess affective responses to touch. It has been used to measure valencespecific reactions to visual ${ }^{23}$, audio-visual ${ }^{24}$, olfactory ${ }^{25}$, and gustatory ${ }^{26}$ stimuli. Facial EMG is a safe and non-invasive method consisting of surface electrodes that adhere to the face ${ }^{27}$. These surface electrodes record facial muscle activity continuously in real-time with time scale sensitivity in the tens of milliseconds. Of particular interest is the corrugator supercilii ("corrugator"), which is activated when furrowing the brow and relaxes during a smile. As a result, corrugator activity has a linear relationship with affective valence, with increased response to negative stimuli and decreased activity in response to positive stimuli ${ }^{28}$. In addition, the zygomaticus major ("zygomatic") is the muscle activated as the corners of the mouth pull up into a smile. The zygomatic displays a "J-shaped" activation pattern with positive stimuli eliciting the greatest response, and the most negative stimuli eliciting a greater response than neutral stimuli ${ }^{28}$. Facial EMG recordings of these muscles can even be observed when stimuli are presented outside conscious awareness or when individuals are explicitly trying to suppress their reactions ${ }^{29,30}$. Importantly, facial EMG can be used alone or in combination with self-report ratings or other physiological recordings. Thus, it is an ideal methodology to assess affective reactions to tactile stimulation ${ }^{31,32}$.

In sum, facial EMG can be combined with self-report ratings to determine how CT-optimal tactile stimulation influences facial muscle activity as a potential indicator of affective response. One can take advantage of the velocity-dependent firing frequency of CTs to apply touch at CToptimal and non-optimal velocities, and touch can be applied both to the CT-rich arm and the putatively CT-lacking palm. Comparisons can be made across modalities to determine whether affective responses to touch require direct stimulation or can be elicited via mere observation, suggestive of shared processing across sensory modalities. Finally, upon establishing facial EMG as a suitable methodology to study affective reactions to affective touch, researchers can then explore how affective touch processing may be influenced by various interventions (e.g., drug administration; stress exposure), how it changes throughout development ${ }^{7}$, how it is influenced by the relationship of the interactants ${ }^{33}$, and whether it is dysregulated in clinical populations ${ }^{8}$.

\section{Protocol}

This protocol is based on Mayo et al. ${ }^{31}$ (Experiment 1 ) and Ree et al. ${ }^{32}$ (Experiment 2). Ethical approval was granted by the Regional Ethical Review Board, Linköping, Sweden (Experiment 1) and the local ethical committee at the Department of Psychology, University of Oslo, Norway (Experiment 2).

\section{Participant Screening and Preparation}

1. Recruit participants who lack tactile or uncorrected visual disturbances and are free of any neurological or psychiatric disorder, unless a specific patient population is being recruited.

2. Ensure that participants are fully able to understand task instructions (e.g., fluent in the language that tasks are administered).

3. If including more than one task (e.g. Experienced, Observed), ensure that task order is counterbalanced across participants, stratifying for gender, age, or other distinguishing factors.

\section{Stimuli and Task Construction}

\section{NOTE: See Table 1 for experimental design.}

\section{Experienced touch task (Experiments 1 and 2)}

1. Create trials such that they consist of a baseline period, touch administration, and self-report ratings, all separated by jittered ITIs.

1. Baseline periods consist of a blank screen, fixation cross, or other neutral scene prior to tactile stimulation.

2. Tactile stimulation is followed by a short (e.g. 1-2 s) ITI, then self-report ratings are obtained.

3. A jittered inter-trial interval (ITI; e.g. 6-7 s) follows self-report ratings to allow muscle activity to return to baseline levels before the next trial begins.

2. Use either audio (Experiment $1^{31}$ ) or visual (Experiment $2^{32}$ ) cues to ensure that touch is delivered at the appropriate velocity.

1. To use audio cues, have cues delivered to headphones worn by the experimenter to track the pace of the stimulation using a metronome. Distinguish velocities using tones of differing pitches (or other distinguishing audio cue, e.g., a cue saying "10 cm/s") that precede the stimulation cues.

2. To use visual cues, display cues on a tablet only in view of the experimenter. Use a moving bar to track velocity of touch administration.

3. Prior to the start of the study, practice to ensure that touch is delivered at the appropriate velocity and a consistent pressure. To do so, apply brushstrokes to the scale in a similar manner as to the participant. The scale readout is used to determine if the pressure changes throughout touch administration. For instance, a pressure of $0.4 \mathrm{~N}$ would read as $40 \mathrm{~g}$ on the scale.

2. Observed touch task (Experiment 1)

1. Ensure that videos of touch administration are of similar length, regardless of velocity. 
1. Include both CT-optimal $(1-10 \mathrm{~cm} / \mathrm{s})$ and non-optimal (less than $1 \mathrm{~cm} / \mathrm{s}$ or more than $10 \mathrm{~cm} / \mathrm{s}$ ) velocities.

2. Start trials with a fixation cross or other neutral condition followed by video.

NOTE: Videos contain touch delivered to CT-rich hairy skin (arm), CT-lacking glabrous skin (palm), and a non-social condition in which touch is delivered to a fake wooden arm (Fig. 2; see supplemental videos).

1. After a 1-2 s ITI, obtain self-report ratings.

2. Allow another 6-7 ITI following ratings to precede the next trial to allow EMG activity to return to baseline.

\section{Facial Electromyography}

1. Data acquisition and filtering guidelines (based on previous protocols ${ }^{27,34}$ )

1. Use software to apply filtering steps either in real-time or offline. Typical filtering steps include a comb band stop filter to filter out potential noise from AC power $(50 / 60 \mathrm{~Hz})$, followed by smoothing and rectification.

NOTE: Initial basic filtering steps may be set on EMG amplifiers (e.g., a high pass filter of $10 \mathrm{~Hz}$ and a low pass filter of $500 \mathrm{~Hz}$ or $1,000 \mathrm{~Hz})$.

2. Electrode application (based on previous protocols ${ }^{27,34}$ )

1. Briefly describe the application process to the participant. Use neutral words ("sensor") instead of potentially anxiety-evoking words $(\text { "electrode") })^{34}$.

1. Decide what information to tell the participants regarding the purpose of the sensors.

NOTE: In the current studies, participants were told sensors would measure muscle and sweat activity during the session.

2. Clean the participants' skin prior to electrode application.

1. Use water to wipe clean the areas in which sensors will be applied.

2. Use an exfoliant scrub to lightly abrade the same areas. Use caution to prevent major skin irritation, though minor irritation is likely to occur.

3. Use electrode pairs consisting of two $4 \mathrm{~mm}$ shielded bipolar recording electrodes plus one monopolar reference electrode.

1. Apply adhesive collars to the electrodes such that they adhere to the skin.

2. Once collars adhere to the outer rim of the electrodes, fill sensors with a conductive electrode gel, taking care to prevent the formation of air bubbles.

4. Place electrode pairs parallel to the muscle(s) of interest and perpendicular to potential sources of noise, such as other muscles ${ }^{34}$.

1. Corrugator: Affix one electrode directly above the eyebrow along an imaginary vertical line that traverses the inner corner of the eye. Place the second electrode $1 \mathrm{~cm}$ lateral and slightly superior to the first, along the border of the eyebrow.

2. Zygomatic: Place the first sensor midway along an imaginary line that connects the upper ear (where the ear meets the skull) and the corner of the mouth. Place the second electrode $1 \mathrm{~cm}$ medial (towards the mouth). Take care to avoid the masseter muscle.

3. Use an $8 \mathrm{~mm}$ unshielded, monopolar recording electrode as a reference electrode. Place the electrode in the middle of the forehead, equidistant (above) the inner brows and (below) the hairline.

4. Ensure that electrode wires are placed such that they do not impede vision. Use medical tape to ensure long-term adherence of the electrodes to skin and reduce noise/artifacts due to cord movement.

5. Determine the quality of electrode application with an impedance monitor. Acceptable impedance levels are below $20 \mathrm{k} \Omega$. If electrodes need to be reapplied to reach appropriate impedance levels, use a clean pair of electrodes.

\section{Task Procedure}

1. General order

1. Following sensor application, complete task(s). If using more than one task, counterbalance order across participants.

2. Ensure that participants are seated comfortably to minimize extraneous movement that may introduce movement artifacts ${ }^{34}$.

2. Experienced touch task

1. Seat participants in front of computer with the to-be-touched arm extended laterally, resting comfortably (e.g., on a cushion). NOTE: It is recommended to apply touch to the arm that is not being used for self-reported ratings in order to minimize potential movement artifacts in the EMG signal.

2. Occlude view of the arm from the participant either using a curtain separator ${ }^{31}$ or goggles that occlude lateral vision (Figure $\left.1^{32}\right)^{35}$.

3. Instruct the participant to focus on how the touch makes them feel.

4. Vary touch location to avoid CT fatigue ${ }^{36}$.

5. Administer touch using a $75 \mathrm{~mm}$ goat hair brush applied to designated section(s) marked on the arm (and palm). Alternatively, apply touch using a force-controlled robot $^{37}$.

6. Use consistent touch administration direction, e.g., back-and-forth (distal-to-proximal, then proximal to distal) or single direction (proximal-to-distal only)

\section{Observed touch task}

1. Seat participant in front of the computer that will display the videos.

2. Instruct the participant that they will have to rate how the video made them feel. 
3. Ensure that the participant is out of view of the experimenter ${ }^{34}$.

\section{Data Cleaning and Analysis}

1. To assess the mean EMG activation to a specific touch stimulus type, compare the response to the touch stimulus to the preceding baseline, i.e. [mean activation during $6 \mathrm{~s}$ touch stimulation] - [mean activation during $1 \mathrm{~s}$ prestimulus "baseline"], as suggested by Fridlund and Cacioppo ${ }^{34}$.

1. Average responses for each touch stimulus type (CT-optimal, non-optimal and, if appropriate, each location (arm/palm).

2. Do this for each muscle (corrugator, zygomatic) and self-report rating (pleasantness, intensity) individually.

2. To obtain a more sensitive time course, compute mean EMG activation during smaller time intervals (e.g., 700 ms; see Figure $5^{32}$ ). Subtract the same $1 \mathrm{~s}$ baseline from all intervals to remove baseline EMG activity.

NOTE: Prior to analysis, it is recommended to have data manually checked by raters blinded to touch conditions to eliminate trials with artifactual activations ${ }^{34}$.

\section{Representative Results}

CT-optimal touch elicits distinct EMG responses compared to fast non-optimal touch across modalities

The first experiment addressed whether differential EMG reactivity could be detected in response to CT-optimal $(3 \mathrm{~cm} / \mathrm{s})$ and fast non-optimal $(30$ $\mathrm{cm} / \mathrm{s}$ ) tactile stimulation that was directly experienced (Figure 3 ) or merely observed (Figure 2 and Figure 3 ) $^{31}$.

Experienced CT-optimal touch was rated as more pleasant than non-optimal touch $(F(1,28)=32.2 ; p<0.001$; Figure $3 \mathbf{A})$ regardless of touch location $(p=0.063$; velocity $\times$ location: $p=0.32$ ). Similarly, observed CT-optimal touch was rated as more pleasant that non-optimal touch (touch velocity: $F(1,28)=47.5 ; p<0.001$; touch type: $F(2,56)=6.09, p=0.004$; type $x$ velocity interaction $F(2,56)=5.87, p=0.005)$. CT-optimal touch to the arm was rated as more pleasant than touch to the palm $(p=0.024)$ and non-social touch (e.g., touch to the wooden arm; $p=0.001)$. Fast non-optimal touch was always rated as more intense (Figure 3B), regardless of whether the touch was experienced (touch velocity: $F(1,28)=$ 34.3, $p<0.001$; touch location: $p=0.28$; velocity $\mathrm{x}$ location interaction: $p=0.64$ ) or observed (touch velocity: $F(1,28)=35.1, p<0.001 ;$ touch type: $p=0.40$; velocity $x$ type interaction: $p=0.39$ ).

Experienced fast, non-optimal touch elicited robust corrugator reactivity that was mitigated by recruitment of CT-afferents during CT-optimal touch (effect of touch velocity: $F(1,28)=4.84, p=0.036$; effect of touch location: $p=0.93$; touch velocity $\mathrm{x}$ location interaction: $p=0.42$; Figure 3C). Corrugator response significantly differed between CT-optimal and non-optimal touch for touch to the arm $(p=0.050)$ but only trend level effects were seen for touch to the palm $(p=0.092)$. There was no main effect of touch velocity $(p=0.11)$ or type $(p=0.79)$ on corrugator reactivity to observed touch, but there was a touch velocity $x$ type interaction $(F(2,56)=3.80, p=0.028)$. Post hoc tests revealed that fast nonoptimal touch elicited greater corrugator reactivity than CT-optimal touch particularly for videos of touch to the arm $(p=0.007)$, but not touch to the palm $(p=0.13)$ or non-social touch $(p=0.25)$. Zygomatic activity was not significantly affected by experienced touch (effect of touch velocity: $p=0.15$; effect of touch type: $p=0.73$; touch velocity $\mathrm{x}$ type interaction: $p=0.63$; Figure 3D), nor observed touch (main effect of touch velocity: $p$ $=0.37$; main effect of touch type: $p=0.84$; touch velocity $x$ type interaction: $p=0.23$ )

\section{CT-optimal touch elicits EMG responses distinct from slow non-optimal touch}

Experiment 2 assessed whether slow non-optimal $(0.3 \mathrm{~cm} / \mathrm{s})$ would elicit similar responses as fast non-optimal $(30 \mathrm{~cm} / \mathrm{s})^{32}$. We found that slow non-optimal touch was rated as less pleasant (Figure 4A) and less intense (Figure 4B) than CT-optimal touch. Similar to fast non-optimal touch, slow non-optimal touch elicited robust corrugator activitythat was attenuated by CT-optimal touch (effect of touch velocity: $F(1,83)=9.723, p=$ 0.002; Figure 4C). There was no effect of touch on zygomatic activity ( $p=0.35$; Figure 4D).

We next assessed the time course of EMG responses. During the first $700 \mathrm{~ms}$, a window putatively free of $C T$ input, there was no difference in corrugator reactivity $\left(-0.031 \pm 0.06 \mu \mathrm{V}\right.$ and $-0.017 \pm 0.49 \mu \mathrm{V}, p_{B o n}=0.98$; Figure $\left.5 \mathrm{~A}\right)$. However, over the next $5.6 \mathrm{~s}$, corrugator reactivity in response to CT optimal touch decreased gradually, whereas it gradually increased in response to slow non-optimal touch: during interval 2 , the corrugator reactivity was marginally lower for CT optimal touch than non-optimal touch $\left(p_{B o n}=0.071\right)$. During intervals $3,5,6,7$ and 8 , the corrugator reactivity was significantly lower during CT optimal touch than during non-optimal touch $\left(p_{B o n}<0.034\right.$; Figure $\left.5 \mathbf{A}\right)$. This pattern was absent in analysis of zygomatic reactivity ( $p=0.83$; Figure 5B). 


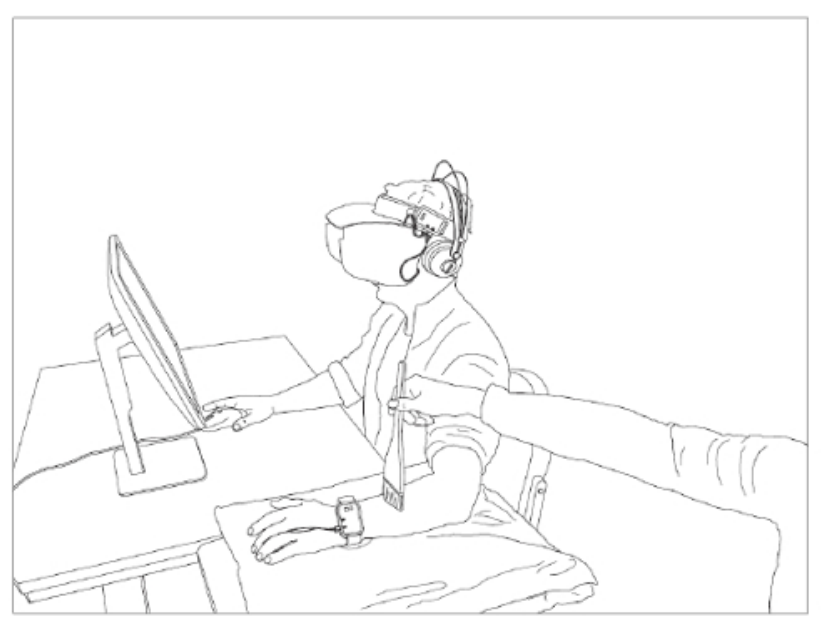

Figure 1: Example of experimental setup for the Experienced Touch task. Seat the participant in front of the computer with their arm extended laterally, comfortably resting on a cushion. If obtaining self-report ratings, it is recommended to apply touch to the arm that is not used to provide ratings to avoid potential movement artifacts from contaminating the EMG signal. The arm should be occluded from view of the participant ${ }^{35,39}$, either with customized glasses, as above, or using a curtain separator. This figure is adapted from Ree et al. ${ }^{32}$ Please click here to view a larger version of this figure.
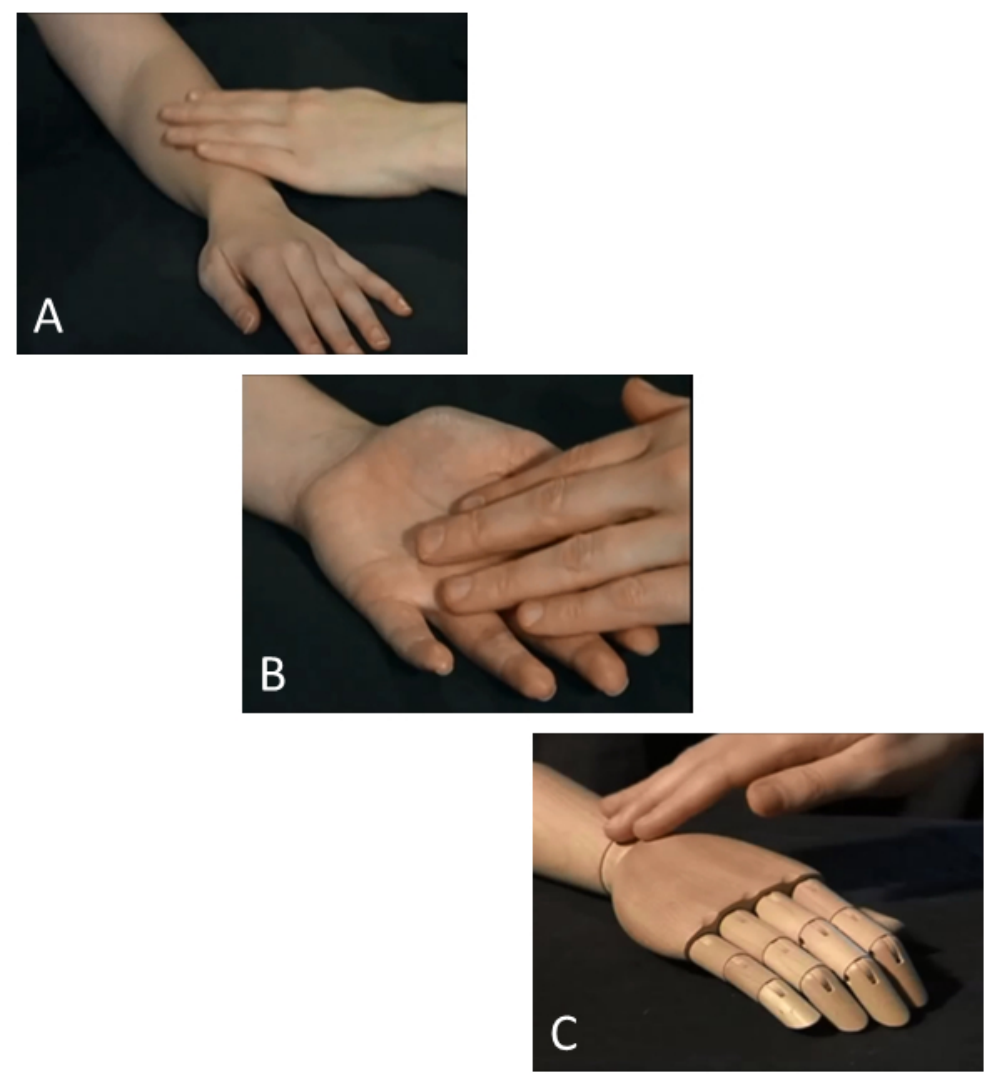

Figure 2: Example of touch stimuli used in the Observed Touch task. The observed touch task included $6 \mathrm{~s}$ videos of touch to the (A) CTrich arm, (B) CT-lacking palm, and (C) non-social touch to a wooden arm. Please click here to view a larger version of this figure. 

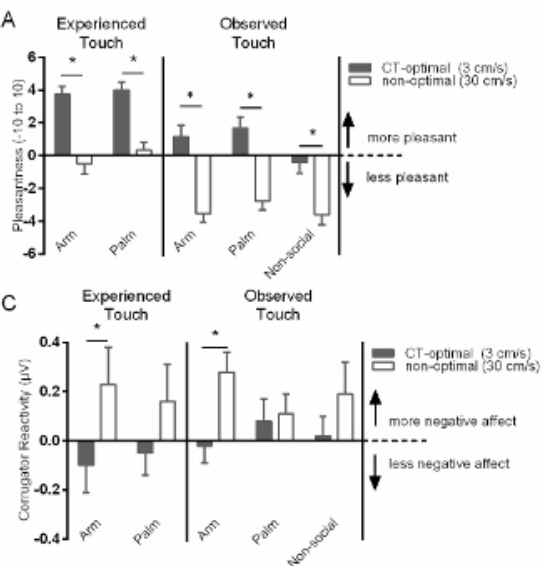
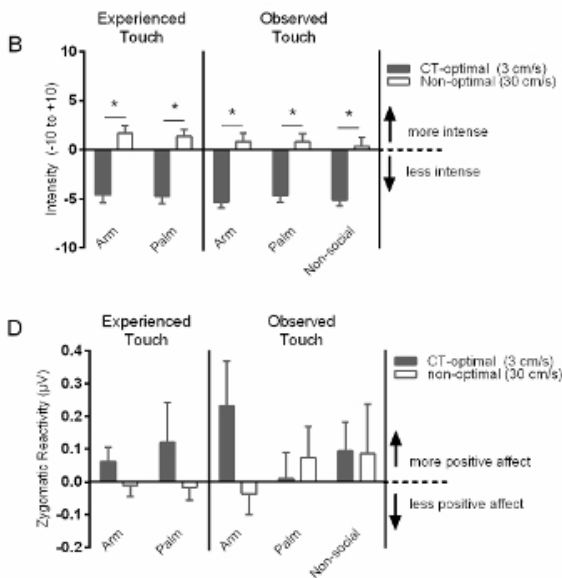

Figure 3: CT-optimal touch elicits distinct responses compared to fast non-optimal touch across modalities. (A) CT-optimal touch (3 cm/ s) is consistently rated as more pleasant than fast non-optimal touch $(30 \mathrm{~cm} / \mathrm{s})$ across both tasks. Experienced touch is rated as most pleasant, followed by social (arm, palm) observed touch, then non-social touch (e.g. touch to a wooden arm). (B) CT-optimal touch is $(3 \mathrm{~cm} / \mathrm{s})$ rated as less intense across modalities, regardless of modality or social content. (C) Fast non-optimal touch $(30 \mathrm{~cm} / \mathrm{s})$ elicits more corrugator reactivity than CT-optimal touch $(3 \mathrm{~cm} / \mathrm{s})$. This difference is most robust for touch to the CT-rich arm. (D) CT-optimal touch $(3 \mathrm{~cm} / \mathrm{s})$ marginally increases zygomatic reactivity, though this does not reach significance for any modality or location. Bars and errors bars represent mean and standard error of the mean; ${ }^{*} p<0.05$ effect of velocity. This figure is adapted from Mayo et al. ${ }^{31}$ Please click here to view a larger version of this figure.

A

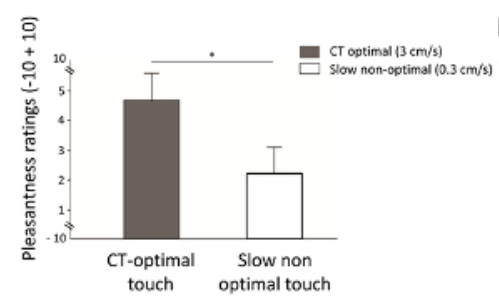

C

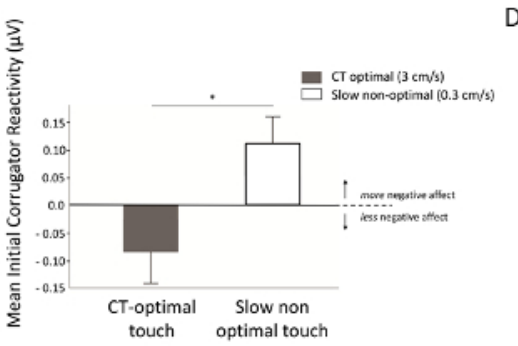

B

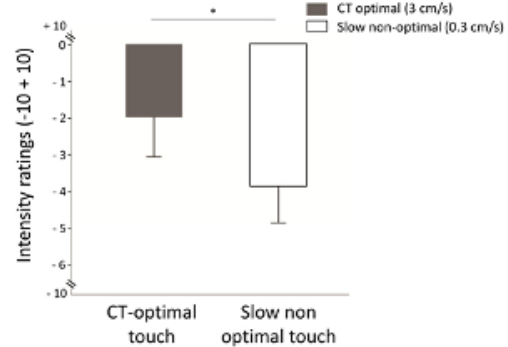

政

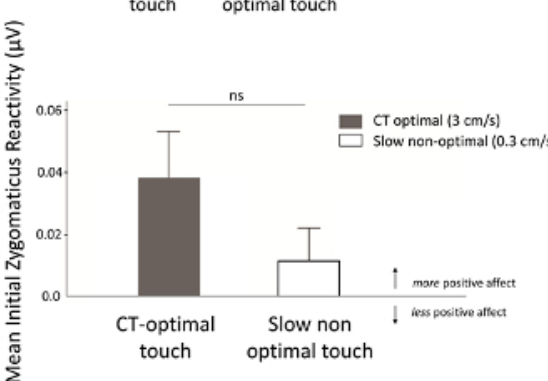

Figure 4: CT-optimal touch $(3 \mathrm{~cm} / \mathrm{s})$ elicits distinct responses as compared to slow non-optimal touch $(0.3 \mathrm{~cm} / \mathrm{s})$. (A) CT-optimal touch (3 $\mathrm{cm} / \mathrm{s})$ is rated as more pleasant than slow non-optimal touch $(0.3 \mathrm{~cm} / \mathrm{s})$. (B) CT-optimal touch $(3 \mathrm{~cm} / \mathrm{s})$ is rated as more intense than slow nonoptimal touch $(0.3 \mathrm{~cm} / \mathrm{s})$. (C) Mean corrugator reactivity in response to CT-optimal $(3 \mathrm{~cm} / \mathrm{s})$ is reduced compared to slow non-optimal $(0.3 \mathrm{~cm} / \mathrm{s})$. (D) Touch does not significantly influence zygomatic reactivity. Bars and error bars represent means and standard error of the mean; ${ }^{*} p<0.05$. This figure is adapted from Ree et al. ${ }^{32}$ Please click here to view a larger version of this figure. 

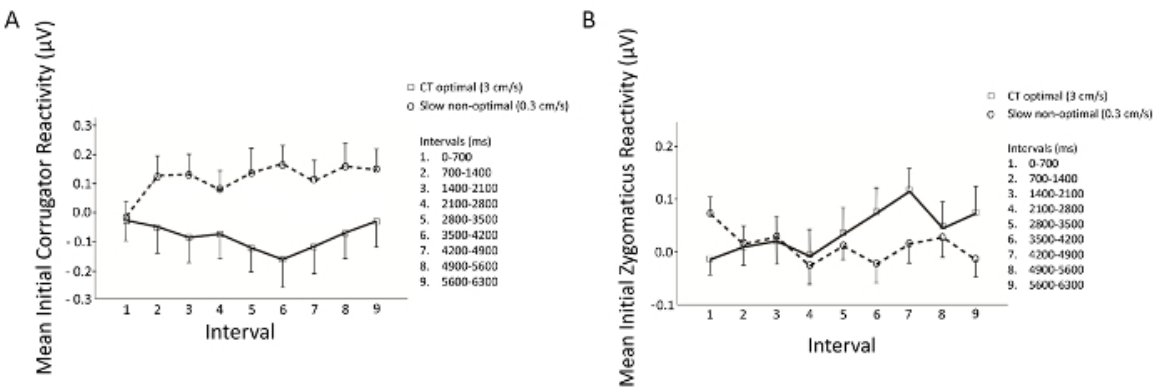

Figure 5: Corrugator responses to CT-optimal touch are temporally specific. (A) When binned in intervals of $700 \mathrm{~ms}$, CT-optimal touch elicits significantly less corrugator reactivity. The exception is in the first $700 \mathrm{~ms}$, which is putatively free of CT input due to the slower conduction velocity of these unmyelinated afferents. (B) Zygomatic reactivity is not significantly different in response to optimal or slow non-optimal touch at any of the time points. Dots represent means and bars represent standard errors of the mean. This figure is adapted from Ree et al. ${ }^{32}$ Please click here to view a larger version of this figure.

\begin{tabular}{|c|c|c|c|c|c|c|}
\hline & Study N & $\begin{array}{l}\text { Velo } \\
\end{array}$ & city & Touch & Touch & Number of \\
\hline & $(M / F)$ & CT-optimal & $\begin{array}{c}\text { Non- } \\
\text { optimal }\end{array}$ & Location/Type & Duration & repetitions \\
\hline \multicolumn{7}{|l|}{ Experiment 1} \\
\hline Experienced Touch & $30(17 / 13)$ & $3 \mathrm{~cm} / \mathrm{s}$ & $30 \mathrm{~cm} / \mathrm{s}$ & Arm, palm & $6 \mathrm{sec}$ & $\begin{array}{c}8 \times \text { velocity, location } \\
\text { (32 total) }\end{array}$ \\
\hline Observed Touch & $30(17 / 13)$ & $3 \mathrm{~cm} / \mathrm{s}$ & $30 \mathrm{~cm} / \mathrm{s}$ & $\begin{array}{l}\text { Arm, palm, non- } \\
\text { social }\end{array}$ & $6 \mathrm{sec}$ & $\begin{array}{c}4 \times \text { location, type } \\
\text { total) }\end{array}$ \\
\hline \multicolumn{7}{|l|}{ Experiment 2} \\
\hline Experienced Touch & $\begin{array}{c}44 \\
(17 / 27) \\
\end{array}$ & $3 \mathrm{~cm} / \mathrm{s}$ & $0.3 \mathrm{~cm} / \mathrm{s}$ & Arm & $\begin{array}{c}6.3 \mathrm{sec} \\
\text { (of } 2 \mathrm{~min} \text { ) } \\
\end{array}$ & $\begin{array}{r}2 \times \text { velocity } \\
\text { total) }\end{array}$ \\
\hline
\end{tabular}

Table 1: Summary of Experimental Designs. In the Experienced Touch task of Experiment 1, touch was delivered at CT-optimal (3 cm/s) or fast non-optimal $(30 \mathrm{~cm} / \mathrm{s})$ velocities to hairy (arm) and glabrous (palm) skin. The Observed Touch instead included videos of touch delivered to the arm, palm, or to a wooden arm (e.g., non-social) at the same touch velocities. The "non-social" condition was included to control for potential responses elicited by low-level periodicity information encoded in the movement ${ }^{17}$, and determine the relevance of social content ${ }^{38}$ on ratings $^{\circ}$ and EMG responses. Results were analyzed using repeated measures analysis of variance (ANOVA) with touch velocity and touch type as within-subjects factors. A post-hoc power analysis based on Experiment 1 suggests at least 22 individuals should be included to achieve similar effects. In Experiment 2, touch was delivered to the arm at CT-optimal $(3 \mathrm{~cm} / \mathrm{s})$ or slow non-optimal $(0.3 \mathrm{~cm} / \mathrm{s})$ velocities. Touch was delivered for a total of $2 \mathrm{~min}$, but here we only report on the first $6.3 \mathrm{~s}$ in order to compare results to Experiment 1 . Each velocity was repeated twice. In all experiments, self-reported ratings of the affective quality (e.g., pleasantness) and discriminative aspects (e.g., intensity) were assessed ${ }^{10}$.

\section{Discussion}

Here, we report on the use of facial electromyography (EMG) as a method to study affective responses to observed and experienced touch. Previously, many studies have focused on the use of self-report ratings to characterize the affective quality of touch. Touch that optimally activates CT afferents (e.g., $1-10 \mathrm{~cm} / \mathrm{s}$ ) is consistently rated as more pleasant than either faster or slower touch velocities ${ }^{10}$. In contrast, ratings of intensity seem to track with velocity, with faster touch velocities rated as more intense, likely mediated via $A \beta$ fibers ${ }^{37}$. Using two separate datasets, we show that both fast and slow non-optimal touch elicit robust corrugator reactivity that is attenuated during CT-optimal touch. Thus, we find that touch that is rated as less pleasant (e.g., non-optimal touch) also increases corrugator activity, suggestive of enhanced negative affect. In addition, we find that responses are similar across modalities. That is, both observed and experienced touch elicit similar facial muscle activity. In both modalities, these effects were only significant for touch to the arm, and not the palm or a wooden arm. Thus, while self-reported ratings of experienced and observed affective touch are similar regardless of location (arm, palm), facial EMG only significantly differentiates between touch velocities applied to the CT-rich arm, and not the CT-fiber-lacking palm.

The results further show that the temporal sensitivity of facial EMG yields insight into emotional processing that cannot be obtained solely by selfreport. Namely, we found that corrugator reactivity to CT-optimal touch becomes evident at a timescale that coincides with known conduction velocities of CT afferents ${ }^{1,12}$. Thus, in the initial $700 \mathrm{~ms}$ of touch, which are believed to be dominated by $A \beta$ activation, there is no difference in EMG activation between the two touch velocities. However, the distinction between CT-optimal and non-optimal touch becomes evident following the first $700 \mathrm{~ms}$, consistent with the previously reported temporal time lag of CT-afferents ${ }^{2,12}$. Hence, facial EMG is able to detect changes in affective responses to touch that occur with a temporal specificity that is likely inaccessible via verbal reporting.

Across both studies, we find that CT-optimal and non-optimal touch can be distinguished via corrugator activity. However, we did not find an effect of touch on zygomatic reactivity, which is in contrast to previous reports ${ }^{40}$. One potential reason for the discrepancies between the current data and previous findings include methodological differences such as inclusion of a post-touch period in the analysis. Thus, we stress the importance of methodological considerations such as the length of the touch stimulation and inter-trial intervals when designing these experiments.

There are several factors that should be considered when assessing affective reactions to touch. One potential area of concern is the gender of the experimenter (and thus, toucher) to that of the participant, as well as the relationship, if any, between the two ${ }^{41}$. Moreover, one should ensure that participants are precluded from viewing the experimenter and touch application, as visual processing of touch can influence the perception of touch $^{35,39}$. There are also concerns to weigh during task design. For instance, it is important to consider the potential for order effects, both in 
regards to touch stimuli presentation (e.g. discussed $\mathrm{in}^{42}$ ) or touch location ${ }^{43}$. If several touch repetitions are used, one may want to vary touch location to avoid CT fatigue ${ }^{36}$. Here, we used a brush to apply touch to compare to previous studies ${ }^{17}$, though it is possible that EMG responses may be different using more ecologically valid methods (e.g., touch by hand).

While we believe the use of facial EMG will be of a great benefit to the field of affective touch, there are limitations to this methodology that warrant consideration. Training is required to learn how to apply the electrodes correctly, producing an increased burden on the experimenter on the outset of experimental planning. Excessive movement, talking, or other environmental factors present during the experiment may cause artifacts in the EMG signal, thus constraining some experimental design features. Moreover, the application of electrodes to the face may elicit an attempt to discern the purpose of the study. As such, one must consider what information to tell the participant regarding not only the purpose of the experiment, but also the use of the electrodes during the experiment. In the current experiments, the participants were told that the purpose of the study was the investigate decision-making and perceptions of various sensations ${ }^{32}$ or reactions to social interactions ${ }^{31}$. In both cases, participants were told that the electrodes would measure sweat and muscle activity and were fully debriefed following the conclusion of the experiment. These concerns and others are addressed thoroughly in Fridlund and Cacioppo $1986^{34}$.

In sum, we demonstrate that facial EMG is a reliable, robust, and informative method to assess the affective valence of tactile stimulation. This method provides a means to implicitly assess responses to tactile stimulation independent of verbal reports, paving the way for studies in infants and young children, cross-cultural comparisons, investigations of clinical conditions, and other situations in which semantics and language may otherwise preclude scientific exploration.

\section{Disclosures}

The authors have nothing to disclose

\section{Acknowledgments}

The authors are grateful to Dr. Margaret Wardle for her exceptional training and technical assistance. This work was funded in part by Swedish Research Council grant FYF-2013-687 (IM).

\section{References}

1. Abraira, V. E., Ginty, D. D. The sensory neurons of touch. Neuron. 79 (4), 618-639 (2013).

2. Olausson, H., Wessberg, J., Morrison, I., McGlone, F., Vallbo, A. The neurophysiology of unmyelinated tactile afferents. Neuroscience and Biobehavioral Reviews. 34 (2), 185-191 (2010).

3. Gallace, A., Spence, C. The science of interpersonal touch: an overview. Neuroscience and BiobehavioralReviews. 34 (2), $246-259$ (2010).

4. Morrison, I., Loken, L. S., Olausson, H. The skin as a social organ. Experimental Brain Research. 204 (3), 305-314 (2010).

5. Morrison, I. et al. Reduced C-afferent fibre density affects perceived pleasantness and empathy for touch. Brain: A Journal of Neurology. 134 (Pt 4), 1116-1126 (2011).

6. Olausson, H. et al. Unmyelinated tactile afferents signal touch and project to insular cortex. NatureNeuroscience. 5 (9), $900-904$ (2002).

7. Croy, I., Sehlstedt, I., Wasling, H. B., Ackerley, R., Olausson, H. Gentle touch perception: From early childhood to adolescence. Developmental Cognitive Neuroscience. (2017).

8. Croy, I., Geide, H., Paulus, M., Weidner, K., Olausson, H. Affective touch awareness in mental health and disease relates to autistic traits - An explorative neurophysiological investigation. Psychiatry Research. 245, 491-496 (2016).

9. Crucianelli, L., Cardi, V., Treasure, J., Jenkinson, P. M., Fotopoulou, A. The perception of affective touch in anorexia nervosa. Psychiatry Research. 239, 72-78 (2016).

10. Loken, L. S., Wessberg, J., Morrison, I., McGlone, F., Olausson, H. Coding of pleasant touch by unmyelinated afferents in humans. Nature Neuroscience. 12 (5), 547-548 (2009).

11. Ackerley, R. et al. Human C-Tactile Afferents Are Tuned to the Temperature of a Skin-Stroking Caress. The Journal of Neuroscience. 34 (8), 2879-2883 (2014)

12. Ackerley, R., Eriksson, E., Wessberg, J. Ultra-late EEG potential evoked by preferential activation of unmyelinated tactile afferents in human hairy skin. Neuroscience Letters. 535, 62-66 (2013).

13. Morrison, I. ALE meta-analysis reveals dissociable networks for affective and discriminative aspects of touch. Human Brain Mapping. 37 (4), 1308-1320 (2016).

14. Case, L. K. et al. Encoding of Touch Intensity But Not Pleasantness in Human Primary Somatosensory Cortex. The Journal of Neuroscience. 36 (21), 5850-5860 (2016).

15. Case, L. K. et al. Touch Perception Altered by Chronic Pain and by Opioid Blockade. eNeuro. 3 (1) (2016).

16. Davidovic, M., Starck, G., Olausson, H. Processing of affective and emotionally neutral tactile stimuli in the insular cortex. Developmental Cognitive Neuroscience. (2017).

17. Morrison, I., Bjornsdotter, M., Olausson, H. Vicarious responses to social touch in posterior insular cortex are tuned to pleasant caressing speeds. The Journal of Neuroscience. 31 (26), 9554-9562 (2011).

18. Nisbett, R. E., Wilson, T. D. Telling more than we can know: Verbal reports on mental processes. Psychological Review. 84 (3), $231-259$ (1977).

19. Sato, H., Kawahara, J. Selective bias in retrospective self-reports of negative mood states. Anxiety, Stress, and Coping. 24 (4), $359-367$ (2011).

20. Robinson, M. D., Clore, G. L. Belief and feeling: evidence for an accessibility model of emotional self-report. Psychological Bulletin. 128 (6), 934-960 (2002).

21. Cascio, C. J. et al. Perceptual and neural response to affective tactile texture stimulation in adults with autism spectrum disorders. Autism Research. 5 (4), 231-244 (2012). 
22. Tager-Flusberg, H., Paul, R., Lord, C. Language and communication in autism. Handbook of Autism and Pervasive Developmental Disorders. 1, 335-364 (2005).

23. Lang, P. J., Greenwald, M. K., Bradley, M. M., Hamm, A. O. Looking at pictures: affective, facial, visceral, and behavioral reactions. Psychophysiology. 30 (3), 261-273 (1993).

24. Rozga, A., King, T. Z., Vuduc, R. W., Robins, D. L. Undifferentiated facial electromyography responses to dynamic, audio-visual emotion displays in individuals with autism spectrum disorders. DevelopmentalScience. 16 (4), 499-514 (2013).

25. Joussain, P., Ferdenzi, C., Djordjevic, J., Bensafi, M. Relationship Between Psychophysiological Responses to Aversive Odors and Nutritional Status During Normal Aging. ChemicalSenses. 42 (6), 465-472 (2017).

26. Horio, T. EMG activities of facial and chewing muscles of human adults in response to taste stimuli. Perceptual and Motor Skills. 97 (1), 289-298 (2003).

27. Tassinary, L. G., Cacioppo, J. T., Vanman, E. J. in Handbook of Psychophysiology., eds. Gary Berntson, John T. Cacioppo, Louis G. Tassinary. 267-300. Cambridge University Press, Cambridge, UK (2007).

28. Larsen, J. T., Norris, C. J., Cacioppo, J. T. Effects of positive and negative affect on electromyographic activity over zygomaticus major and corrugator supercilii. Psychophysiology. 40 (5), 776-785 (2003).

29. Dimberg, U., Thunberg, M., Grunedal, S. Facial reactions to emotional stimuli: Automatically controlled emotional responses. Cognition and Emotion. 16 (4), 449-471 (2002).

30. Dimberg, U., Thunberg, M., Elmehed, K. Unconscious facial reactions to emotional facial expressions. Psychological Science. 11 (1), $86-89$ (2000).

31. Mayo, L. M., Lindé, J., Olausson, H., Heilig, M., Morrison, I. Putting a good face on touch: Facial expression reflects the affective valence of caress-like touch across modalities. Biological Psychology. (2018).

32. Ree, A., Mayo, L. M., Leknes, S., Sailer, U. Touch targeting C-tactile afferent fibers has a unique physiological pattern: a combined electrodermal and facial electromyography study. Biological Psychology. (2018).

33. Kreuder, A. K. et al. How the brain codes intimacy: The neurobiological substrates of romantic touch. Human Brain Mapping. 38 (9), 4525-4534 (2017).

34. Fridlund, A. J., Cacioppo, J. T. Guidelines for human electromyographic research. Psychophysiology. 23 (5), $567-589$ (1986).

35. Tipper, S. P. et al. Vision influences tactile perception without proprioceptive orienting. Neuroreport. 9 (8), 1741-1744 (1998).

36. Vallbo, A. B., Olausson, H., Wessberg, J. Unmyelinated Afferents Constitute a Second System Coding Tactile Stimuli of the Human Hairy Skin. Journal of Neurophysiology. 81 (6), 2753-2763 (1999).

37. Triscoli, C., Olausson, H., Sailer, U., Ignell, H., Croy, I. CT-optimized skin stroking delivered by hand or robot is comparable. Frontiers in Behavioral Neuroscience. 7, 208 (2013).

38. Croy, I. et al. Interpersonal stroking touch is targeted to C tactile afferent activation. Behavioural Brain Research. 297, 37-40 (2016).

39. Keizer, A., de Jong, J. R., Bartlema, L., Dijkerman, C. Visual perception of the arm manipulates the experienced pleasantness of touch. Developmental Cognitive Neuroscience. (2017).

40. Pawling, R., Cannon, P. R., McGlone, F. P., Walker, S. C. C-tactile afferent stimulating touch carries a positive affective value. PloS One. 12 (3), e0173457 (2017).

41. Scheele, D. et al. An oxytocin-induced facilitation of neural and emotional responses to social touch correlates inversely with autism traits. Neuropsychopharmacology. 39 (9), 2078-2085 (2014).

42. Ackerley, R., Saar, K., McGlone, F., Backlund Wasling, H. Quantifying the sensory and emotional perception of touch: differences between glabrous and hairy skin. Frontiers in Behavioral Neuroscience. 8 (34) (2014).

43. Loken, L. S., Evert, M., Wessberg, J. Pleasantness of touch in human glabrous and hairy skin: order effects on affective ratings. Brain Research. 1417, 9-15 (2011). 